\title{
NUCLEAR STARBURSTS IN BARRED SPIRALS
}

\author{
N.A. DEVEREUX \\ Dept. of Physics and Astronomy \\ University of Massachusetts \\ Graduate Research Center \\ Amherst, MA 01002.
}

\section{Introduction}

The first analysis of the Infrared Astronomical Satellite (IRAS) data revealed differences between barred and unbarred spirals. The study of Shapley-Ames galaxies, by De Jong et al (1984), revealed that barred spirals tend, on average, to be associated with "warmer" far infrared color temperatures and higher $\mathrm{L}_{\text {fir }} / \mathrm{L}_{\text {blue }}$ ratios than unbarred spirals. In a related study Hawarden et al (1986) noted that barred and unbarred spirals could be partially segregated on the basis of the IRAS $\mathrm{S}_{25 \mu \mathrm{m}} / \mathrm{S}_{12 \mu \mathrm{m}}$ flux ratio. Both sets of investigators attributed the differences, between barred and unbarred spirals, to a nuclear starburst in the barred types.

Unfortunately, the IRAS data alone can not be used to separate the nuclear and disk contribution to the total emission owing to the low angular resolution of the satellite telescope. To overcome this limitation ground based $10 \mu \mathrm{m}$ measurements of the central $5 "$ of spirals were obtained. The combination of the ground based and IRAS data has provided a first order estimate of the spatial distribution of infrared emission by establishing the fraction of the "total" IRAS emission that is radiated within the central 5". Ground based measurements have been obtained for 150 Shapley-Ames type spiral galaxies and the large database has permitted a comprehensive statistical analysis of the infrared morphology of spirals which exhibit a wide range of optical morphology.

\section{Sample and Analysis}

In the interest of brevity the reader is referred to Devereux (1987) for a full discussion of the sample and analysis. Briefly, the sample includes all known infrared luminous $\mathrm{L}(40-120 \mu \mathrm{m}) \geq 3$ $x 10^{9} \mathrm{~L}$ non-interacting spiral galaxies within the distance range $15 \leq \mathrm{D}(\mathrm{Mpc}) \leq 40$. The data analysis has been carried out with a view to elucidate differences between barred and unbarred spirals. Although the sample was also segregated into Early (Sb and earlier) and Late (Sbc and later) types in order to isolate differences which may depend on bulge/disk ratio rather than the presence of a stellar bar.

\section{Results and Discussion}

Significant differences are found between barred and unbarred Early-type spirals. Approximately half of the barred spirals exhibit central $10 \mu \mathrm{m}$ luminosities which exceed the maximum observed 
for the unbarred spirals. As a result the mean central $10 \mu \mathrm{m}$ luminosity for the barred spirals is approximately 3 times higher than the unbarred spirals. The luminosity enhancement observed for the Early-type spirals is confined to the central $\sim 1 \mathrm{kpc}$ diameter region.

A follow up ground based study (Devereux 1988) has revealed that the energy distribution of the emission, radiated by the central $1 \mathrm{kpc}$ region, rises steeply between $1.65 \mu \mathrm{m}$ and $10 \mu \mathrm{m}$. Such energy distributions are characteristic of starburst nuclei and the association is confirmed for the majority of the objects with published optical spectra. The origin of the high central $10 \mu \mathrm{m}$ luminosity is therefore identified with star formation.

Evidently, the intensity of nuclear star formation activity is elevated in Early-type barred spirals strongly implicating the bar as the cause. The differences between Late-type barred and unbarred spirals are of less statistical significance. This may indicate that stellar bars are less efficient at enhancing the intensity of nuclear star formation activity in Late-types. On the other hand it is also true that a significant percentage $\sim 40 \%$ of all spirals for which the intensity of the starburst activity is comparable to M82 and NGC 253 are classified as Late-type and also exhibit conspicuous stellar bars.

Other studies have demonstrated the frequency of occurrence of nuclear star formation activity to be enhanced in barred spirals (Heckman 1980, Balzano 1983). It is believed, however, that the present study is the first to demonstrate that the intensity of star formation activity is also enhanced in barred spirals.

A possible bar induced mechanism which may enhance the intensity of star formation in barred spirals is discussed by Combes and Gerin (1985). The idea emphasizes enhanced star formation through cloud-cloud collisions at the site of the bar induced Inner Lindblad Resonance. Such a resonance is expected to manifest observationally as a ring of star formation. The location and indeed the existence of the ring depends on the galaxy mass distribution and the angular velocity of the bar. However, as discussed in more detail elsewhere (Devereux 1987) the resonance and its associated star formation activity is expected to be close to the nucleus in Early-type spirals. The idea is particularly attractive in view of the finding that the differences between barred and unbarred spirals are most apparent in Early spiral types.

\section{Conclusion}

The maximum intensity of star formation activity is higher in the central $\sim 1 \mathrm{kpc}$ of Early-type barred spirals than the central $\sim 1 \mathrm{kpc}$ of Early-type unbarred spirals. The bar is implicated as the cause for the difference.

\section{References}

Balzano, V.A., 1983, ApJ 268, 602.

Combes, F., and Gerin, M., 1985, Astron. Astrophys. 150, 327.

De Jong, T., Clegg, P.E., Soifer, B.T., Rowan-Robinson, M., Habing, H.J., Houck, J.R., Aumann, H.H., and Raimond, E., 1984, ApJ (Letters), 278, L67.

Hawarden, T.G., Mountain, C.M., Leggett, S.K., and Puxley, P.J., 1986, M.N.R.A.S., 221, 41P.

Devereux, N.A., 1987, ApJ 323, 91.

Devereux, N.A., 1988, ApJ (submitted).

Heckman, T.M., 1980, Astron. Astrophys. 88, 365. 